\title{
3D inversion of airborne electromagnetic data using a moving footprint
}

\author{
Leif H. Cox ${ }^{1,2}$ Glenn A. Wilson ${ }^{2,4}$ Michael S. Zhdanov ${ }^{2,3}$ \\ ${ }^{1}$ Department of Geophysical Engineering, Montana Tech, 1300 West Park Street, Butte, \\ MT 59701, USA. \\ ${ }^{2}$ Technolmaging, 4001 South, 700 East, Suite 500, Salt Lake City, UT 84107, USA. \\ ${ }^{3}$ Department of Geology and Geophysics, The University of Utah, 1450 East, 100 South, \\ Salt Lake City, UT 84102, USA. \\ ${ }^{4}$ Corresponding author. Email: glenn@technoimaging.com
}

\begin{abstract}
It is often argued that 3D inversion of entire airborne electromagnetic (AEM) surveys is impractical, and that 1D methods provide the only viable option for quantitative interpretation. However, real geological formations are 3D by nature and $3 \mathrm{D}$ inversion is required to produce accurate images of the subsurface. To that end, we show that it is practical to invert entire AEM surveys to 3D conductivity models with hundreds of thousands if not millions of elements. The key to solving a 3D AEM inversion problem is the application of a moving footprint approach. We have exploited the fact that the area of the footprint of an AEM system is significantly smaller than the area of an AEM survey, and developed a robust 3D inversion method that uses a moving footprint. Our implementation is based on the $3 \mathrm{D}$ integral equation method for computing data and sensitivities, and uses the re-weighted regularised conjugate gradient method for minimising the objective functional. We demonstrate our methodology with the 3D inversion of AEM data acquired for salinity mapping over the Bookpurnong Irrigation District in South Australia. We have inverted 146 line km of RESOLVE data for a 3D conductivity model with $\sim 310000$ elements in 45 min using just five processors of a multi-processor workstation.
\end{abstract}

Key Words: 3D, airborne, Bookpurnong, electromagnetic, footprint, inversion, regularisation, RESOLVE.

\section{Introduction}

Airborne electromagnetic (AEM) data are often interpreted using 1D methods, whether by apparent resistivity transforms (e.g. Huang and Fraser, 2002), conductivity depth transforms (e.g. Wolfgram and Karlik, 1995; Macnae et al., 1998; Fullagar and Reid, 2001), S-inversion (Tartaras et al., 2000; Combrinck, 2008), Zohdy's method (Sattel, 2005), layered earth inversions (e.g. Chen and Raiche, 1998; Farquharson et al., 2003; Christensen et al., 2010), laterally constrained layered earth inversions (e.g. Auken et al., 2005, Tartaras and Beamish, 2005; Vallée and Smith, 2009; Viezzoli et al., 2009) or holistic inversions (e.g. Brodie and Sambridge, 2006, 2009). Distinct advantages have been suggested for each particular 1D method. However, all 1D methods are approximations to a 3D Earth, and like all approximations, they have limitations on their applicability.

1D methods place the conductivity model beneath the transmitter-receiver pair's midpoint for each transmitterreceiver pair. The well known 'smoke ring' concept (e.g. Nabighian, 1979; Reid and Macnae, 1998) implies that AEM sensitivity is actually offset from the transmitter-receiver pair's midpoint rather than beneath it. As a result, 1D methods recover conductivity models for each transmitter-receiver pair that are spatially misplaced in 3D. 1D conductivity models are often stitched or interpolated in order to produce a pseudo-3D model over the survey area. However, 3D targets often manifest themselves ambiguously within artefacts or distortions in pseudo-3D models. Despite the widespread use of various 1D methods for the interpretation of AEM surveys, it has been demonstrated that they often fail to recover simple 3D targets (e.g. Ellis, 1995, 1998), particularly in those cases where 2D or 3D geological complexity is present (e.g. Raiche et al., 2001; Wilson et al., 2006).

A lot of emphasis has been placed on improving 1D methods over the development of $3 \mathrm{D}$ inversion. Although 3D parameterised inversion methods such as thin sheets embedded in conductive hosts have been successful for certain types of targets (e.g. Wolfgram and Golden, 2001; Zhdanov et al., 2002; Raiche et al., 2006), the routine use of more generalised 3D modelling and inversion methods has yet to be realised. As such, the various $1 \mathrm{D}$ methods have been considered the only practical approach to AEM interpretation.

The primary problem with $3 \mathrm{D}$ modelling is the necessity to solve as many large linear systems of equations as there are transmitter positions in the survey. For 3D inversion, this problem is exacerbated by the need to repeat the whole process for multiple iterations. In case of inversion, Gauss-Newton methods (or their variants) minimise the number of iterations with near-quadratic convergence. However, both the sensitivity matrix and its generalised inverse still need to be computed. Alternatively, the sensitivity matrix (or its products) need to be computed for steepest descent methods or their variants. The steepest descent methods require many more iterations than Gauss-Newton methods. Both methods converge to the same solution.

Various 3D modelling approximations have been introduced, which simplify the non-linear physics of AEM to a series of linear problems in order to make 3D inversion tractable (e.g. Zhdanov and Tartaras, 2002; Zhang, 2003; Zhdanov and Chernyavskiy, 2004; Cox and Zhdanov, 2006, 2007, 2008). Despite their potential, these methods have limited practical 
application due to the approximate nature of the corresponding 3D modelling algorithms. Ellis (2002) and Raiche et al. (2007) introduced rigorous $3 \mathrm{D}$ inversion methods in order to avoid such approximations. Although more complete in their modelling of the physics of AEM, the rigorous methods were limited to the 3D inversion of several hundred stations of AEM data; primarily due to memory limitations of the sensitivity matrix since Ellis (2002) and Raiche et al. (2007) computed the sensitivities for every transmitter position in every element.

It is widely known that AEM data are sensitive to a limited footprint (Liu and Becker, 1990; Beamish, 2003; Reid et al., 2006). The footprint is defined as the lateral extent of the sensitivity of the AEM system. For frequency-domain AEM systems, Liu and Becker (1990) state that the footprints of the horizontal coplanar and vertical coaxial components are $3.75 \mathrm{~h}$ and $1.35 h$, respectively, where $h$ is the flight height of the transmitter. Liu and Becker (1990) modelled the footprint size at the inductive limit. Reid et al. (2006) showed that the footprints may be as high as 10 times the flight height for low induction numbers. This limits the footprint to less than $400 \mathrm{~m}$ for a typical frequency-domain AEM survey. This is significantly smaller than the area of an AEM survey.

The use of a moving footprint allows for the $3 \mathrm{D}$ inversion of only those parts of the entire $3 \mathrm{D}$ model which are within the footprint of a particular transmitter-receiver pair. The sensitivity matrix for the entire 3D model is constructed as the footprints for all transmitter-receiver pairs are superimposed over the entire 3D model. Brodie and Sambridge $(2006,2009)$ implemented a variation of this approach in their holistic inversion method. They extracted 1D models for each transmitter-receiver pair from spline coefficients describing the 3D layered earth model. The sensitivities for the spline coefficients were computed for the 1D models within the AEM system's footprint and stored in a sparse sensitivity matrix. Brodie and Sambridge (2006, 2009) invert the data to obtain data calibration and processing factors simultaneously modifying both the measured data and model so that each satisfies the other.

While holistic inversion represents progress in the direction of $3 \mathrm{D}$ inversion, the modelled fields and sensitivities are still approximated by 1D modelling. Independently, Cox and Zhdanov $(2006,2007,2008)$ used a similar footprint approach for 3D inversion. Their 3D modelling and inversion was based on the localised quasi-linear integral equation method. In their case, the implementation of the moving footprint replaced the full matrices of both forward and inverse operators with sparse matrices.

Wilson et al. (2010) introduced a moving footprint for full 3D AEM inversion that does not rely on approximations in the modelling or inversion kernels. This paper provides a more detailed account of the methodology. We base our modelling on the 3D contraction integral equation method (Hursán and Zhdanov, 2002). We use a regularised conjugate gradient method for minimising our objective functional (Zhdanov, 2002), which makes it practical to invert tens of thousands of stations of AEM data to models with hundreds of thousands if not millions of elements within hours using a single workstation. We demonstrate the effectiveness of this approach by inverting data from an entire RESOLVE survey acquired for salinity mapping over the Bookpurnong Irrigation District in South Australia. We chose to present this particular case study because Bookpurnong is the nearest representation of 1D geology possible. However, the presence of the Murray River and its associated freshwater, groundwater and irrigation hydrogeology leads to a 3D subsurface conductivity distribution. As the results indicate, 3D interpretation of the
RESOLVE data would be more adequate. In the past, the Bookpurnong area has been extensively studied using a variety of 1D inversion methods, including conductivity depth transforms, layered earth inversion, laterally constrained inversion and holistic inversion. For that reason, we believe that this particular case study provides the best opportunity to make a fair comparison of our 3D inversion results and a variety of 1D methods, unlike the results for more complex 3D terranes. Given vested interests pertaining to geological interpretation of AEM data, we will leave it for the reader to decide whether 3D inversion is an improvement when compared to various 1D methods.

\section{Inversion methodology}

\section{Modelling}

All modelling presented in this paper is based on the $3 \mathrm{D}$ volume integral equation method (e.g. Raiche, 1974; Hohmann, 1975; Weidelt, 1975; Xiong, 1992), whereby the 3D conductivity model is separated into background $\left(\sigma_{b}\right)$ and anomalous $(\Delta \sigma)$ parts:

$$
\sigma(\mathbf{r})=\sigma_{b}(z)+\Delta \sigma(\mathbf{r}) .
$$

The advantage of the $3 \mathrm{D}$ volume integral equation method is that the entire 3D Earth model need not be discretised. Rather, an appropriate background conductivity model is chosen and only the volume of interest containing anomalous conductivity needs to be discretised. This is unlike finite-difference or finite-element methods which require large-scale discretisation and an appropriate choice of boundary conditions so as to emulate an unbound 3D Earth model.

The time-harmonic background $(b)$ electric and magnetic fields are defined as the solutions of the fields due to an external magnetic source over the horizontally layered background model:

$$
\begin{gathered}
\nabla \times \nabla \times \mathbf{E}^{b}(\mathbf{r})+i \omega \mu_{\mathrm{o}} \sigma_{b}(z) \mathbf{E}^{b}(\mathbf{r})=-i \omega \mu_{\mathrm{o}} \nabla \times \mathbf{M}(\mathbf{r}), \\
\nabla \times \nabla \times \mathbf{H}^{b}(\mathbf{r})+i \omega \mu_{\mathrm{o}} \sigma_{b}(z) \mathbf{H}^{b}(\mathbf{r})=-i \omega \mu_{\mathrm{o}} \mathbf{M}(\mathbf{r}),
\end{gathered}
$$

where $\mathbf{M}$ is the extraneous magnetic dipole moment per unit volume. These background fields are computed semi-analytically (e.g. Zhdanov, 2009). The scattered (s) electric fields are defined as the difference between the total and background electric fields; or

$$
\mathbf{E}^{\mathrm{s}}(\mathbf{r})=\mathbf{E}(\mathbf{r})-\mathbf{E}^{b}(\mathbf{r}) .
$$

We can write an integral equation for the scattered electric fields:

$$
\mathbf{E}^{s}\left(\mathbf{r}^{\prime}\right)=\int_{v} \hat{\mathbf{G}}_{\mathrm{E}}\left(\mathbf{r}^{\prime}, \mathbf{r}\right) \cdot \Delta \sigma(\mathbf{r})\left[\mathbf{E}^{b}(\mathbf{r})+\mathbf{E}^{s}(\mathbf{r})\right] \mathrm{d}^{3} r,
$$

where $\hat{\mathbf{G}}_{\mathrm{E}}\left(\mathbf{r}^{\prime}, \mathbf{r}\right)$ is the electric Green's tensor for the background conductivity model. The integration is evaluated over those volumes of interest where the total conductivity differs from the background conductivity. Using the method of moments, equation 5 can be reduced to the linear system:

$$
\mathbf{E}^{s}=(\mathbf{I}-\boldsymbol{\Gamma} \cdot \boldsymbol{\Delta} \boldsymbol{\sigma})^{-1} \cdot \boldsymbol{\Gamma} \cdot \boldsymbol{\Delta} \boldsymbol{\sigma} \cdot \mathbf{E}^{b},
$$

where $\mathbf{E}^{s}$ is the vector of basis function coefficients for the scattered electric field, $\mathbf{I}$ is the identity matrix, $\boldsymbol{\Gamma}$ is the matrix of volume integrated Green's functions for the background conductivity model, and $\Delta \boldsymbol{\sigma}$ is a diagonal matrix of anomalous conductivities. Following Hursán and Zhdanov (2002), we exploit the Toeplitz structure of the large, dense matrix system described by equation 6 . We solve the system using an iterative 
method where fast matrix-vector multiplications are provided by 2D FFT convolutions that reduce storage and complexity. Our implementation of the integral equation method restricts the elements to conform to a uniform horizontal grid with arbitrary vertical discretisation; however, that restriction is not problematic for our intended purpose of 3D inversion.

For 3D AEM modelling, it has been argued that direct solvers are more efficient to implement since they enable multiple right hand side source vectors to be solved simultaneously (e.g. Raiche et al., 2007). This argument is correct only when the system matrix is invariant. For a moving footprint, the system matrix varies from station to station as the anomalous conductivities vary, and thus it requires a new solution for each transmitterreceiver pair. Conveniently, the volume integrated Green's functions are identical and can be translated so they need not be recomputed. Hence, it becomes more efficient to implement an iterative solver for equation 6 . We have chosen the complex generalised minimum residual method (CGMRES), as this has been proven to always converge (Zhdanov, 2002). Moreover, exploiting 2D FFT convolutions for matrix-vector multiplications in the CGMRES algorithm reduces the computational complexity from $O\left(n^{2}\right)$ to $O(n \log n)$.

The scattered magnetic fields are then computed from the equation:

$$
\mathbf{H}^{S}\left(\mathbf{r}^{\prime}\right)=\int_{v} \hat{\mathbf{G}}_{H}\left(\mathbf{r}^{\prime}, \mathbf{r}\right) \cdot \Delta \sigma(\mathbf{r})\left[\mathbf{E}^{b}(\mathbf{r})+\mathbf{E}^{s}(\mathbf{r})\right] \mathrm{d}^{3} r,
$$

where $\hat{\mathbf{G}}_{H}\left(\mathbf{r}^{\prime}, \mathbf{r}\right)$ is the magnetic Green's tensor for the background conductivity model. The total magnetic fields are obtained from the summation of the background and scattered magnetic fields.

\section{Inversion}

Inversion strategies for AEM have been previously discussed by Ellis (1995, 1998), and regularised inversion theory is thoroughly detailed in Zhdanov (2002). Here, we don't intend to repeat the prior art of inversion per se, but instead generally discuss our strategy. For example, there are essentially two deterministic strategies to minimise the Tikhonov parametric functional $P^{\alpha}(\boldsymbol{\sigma})$ :

$$
P^{\alpha}(\boldsymbol{\sigma})=\|A(\boldsymbol{\sigma})-\mathbf{d}\|^{2}+\alpha\left\|\boldsymbol{\sigma}-\boldsymbol{\sigma}_{a p r}\right\|^{2} \rightarrow \min ,
$$

where $A$ is the non-linear forward operator, $\boldsymbol{\sigma}$ is the $N_{m}$ length vector of conductivities, $\mathbf{d}$ is the $N_{d}$ length vector of observed data, $\boldsymbol{\sigma}_{a p r}$ is the $N_{m}$ length vector of a priori conductivities, and $\|\ldots\|$ denotes the respective Euclidean norm. Data and model weights can be introduced to equation 8 through data and model weighting matrices. The first term of equation 8 describes the misfit functional between the predicted and observed AEM data. The second term of equation 8 describes the stabilising functional, which in this case is written as a minimum norm stabiliser. The choice of a stabiliser determines the class of the solutions from which a model is sought, and need not be restricted to the so-called 'smooth' stabilisers only. The regularisation parameter $\alpha$ provides a balance (or bias) between the misfit and stabilising functionals. Statements of 'data-driven' or 'model-driven' inversions are misnomers and are simply manifestations of the user's biasing towards either the misfit or stabilising functionals.

One approach to solving equation 8 is with the Gauss-Newton method which updates the vector of conductivities so as to minimise the vector of residual errors using the iterative scheme:

$$
\boldsymbol{\sigma}_{i+1}=\boldsymbol{\sigma}_{i}+\delta \boldsymbol{\sigma}_{i}=\boldsymbol{\sigma}_{i}+k_{i} \mathbf{F}_{i}^{*} \mathbf{r}_{i},
$$

where $k_{i}$ is a step length, $\mathbf{F}_{i}^{*}$ is the generalised inverse of the $N_{d} \times N_{m}$ Fréchet matrix $\mathbf{F}_{i}$ of normalised sensitivities, and $\mathbf{r}_{i}$ is the
$N_{d}$ length vector of the residual fields between the observed and predicted data on the $i$ th iteration. While the number of iterations is minimised since Gauss-Newton methods generally exhibit near-quadratic convergence, there is non-trivial expense in the computation of the generalised inverse of the Fréchet matrix at

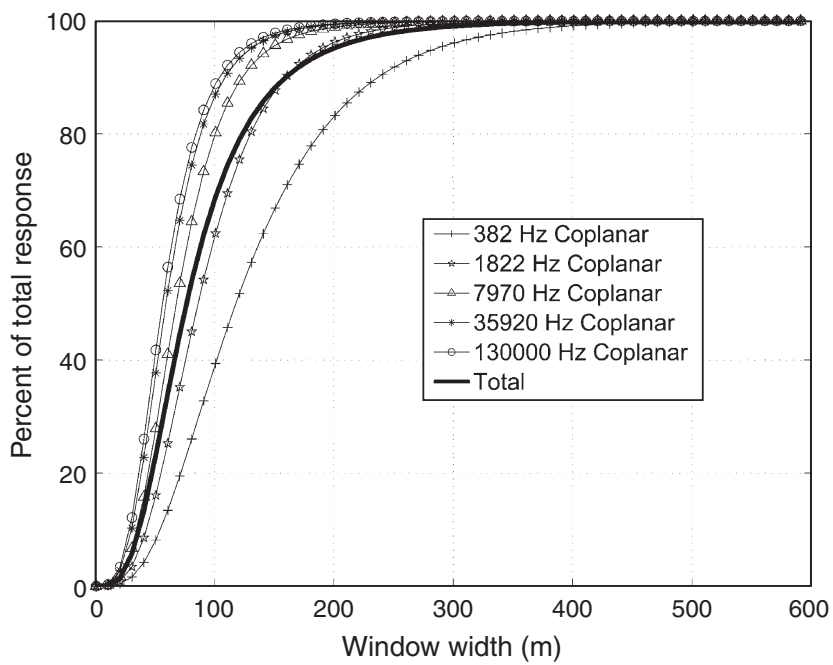

Fig. 1. Total response within a square footprint. The length of one side of the square is given on the abscissa and the percent of the total response given on the ordinate. Over $95 \%$ of the total response comes from within a $200 \mathrm{~m}$ footprint for a $500 \mathrm{mS} / \mathrm{m}$ earth.

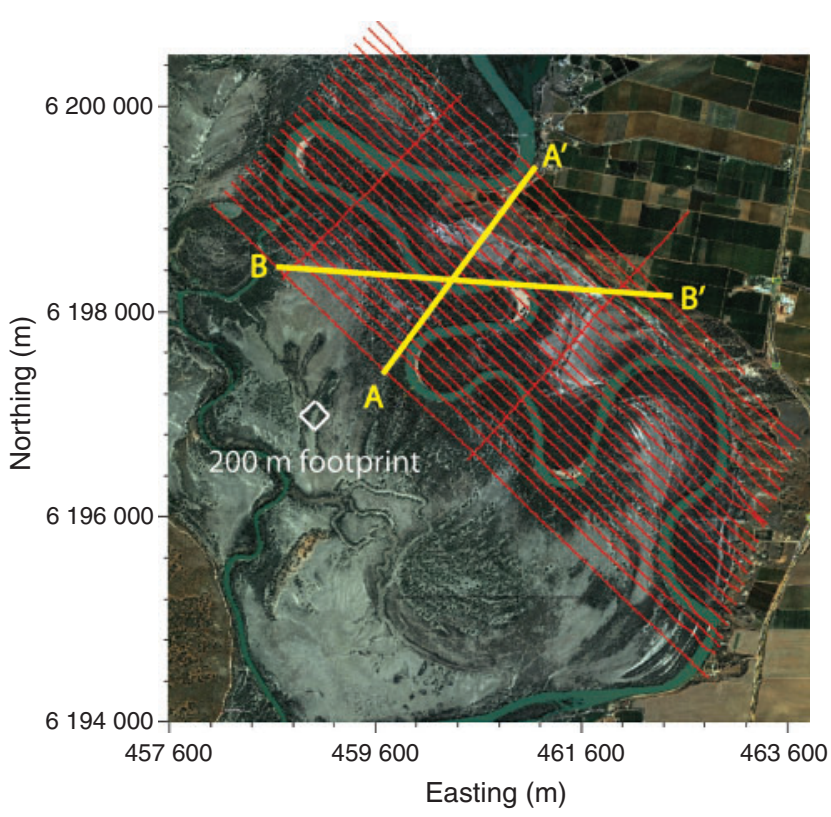

Fig. 2. Satellite image of the Bookpurnong Irrigation District, with RESOLVE flight lines superimposed. Vertical cross-sections from profiles A-A $\mathrm{A}^{\prime}$ and $\mathrm{B}-\mathrm{B}^{\prime}$ are shown in Figs. 6 and 7, respectively. The white square marks the spatial extent of a $200 \mathrm{~m}$ footprint relative to the survey area.

Table 1. Data errors used for 3D inversion.

\begin{tabular}{lc}
\hline Channel & Estimated error (ppm) \\
\hline $390 \mathrm{~Hz}$ Coplanar & 5 \\
$1798 \mathrm{~Hz}$ Coplanar & 5 \\
$8177 \mathrm{~Hz}$ Coplanar & 10 \\
$39460 \mathrm{~Hz}$ Coplanar & 20 \\
$132700 \mathrm{~Hz}$ Coplanar & 25 \\
\hline
\end{tabular}


each iteration. For very large-scale inversions, this is impractical to compute. In addition, a sparse sensitivity matrix will become dense when it is inverted.

An alternative, practical solution for solving equation 8 is with one of the gradient methods which iteratively update the vector of conductivities so as to minimise the vector of residual errors using the iterative scheme:

$$
\boldsymbol{\sigma}_{i+1}=\boldsymbol{\sigma}_{i}+\delta \boldsymbol{\sigma}_{i}=\boldsymbol{\sigma}_{i}+k_{i} \mathbf{F}_{i}^{\mathrm{T}} \mathbf{r}_{i},
$$

where $k_{i}$ is a step length and $\mathbf{F}_{i}^{\mathrm{T}}$ is the conjugate transpose of the Fréchet matrix at the $i$ th iteration. While the number of iterations required to converge upon the same solution is increased compared to Gauss-Newton methods, there is no need to compute the generalised inverse of the Fréchet matrix.

The convergence of the gradient method can be accelerated by including conjugate gradient terms. Our approach has been to use the re-weighted regularised conjugate gradient method (Zhdanov, 2002). Data and model weights, which re-weight the inverse problem in logarithmic space, are introduced so as to reduce the dynamic range of both the data and conductivities. The inversion proceeds to iterate in a manner similar to equation 10 until the residual error reaches a pre-set threshold, the decrease in error between multiple iterations is less than a pre-set threshold, or a maximum number of iterations is reached.

\section{Moving footprint}

At each iteration, computation of the sensitivities for an entire AEM survey and multiplying the transpose of the sensitivity matrix by the residual is not trivial. If one examines the updated model parameters described by equation 10 , the size of $F_{i}{ }^{*}$ is $N_{m} \times N_{d}$. For a small frequency-domain AEM survey, an inversion domain of 250000 model elements and 20000 data points may be reasonably expected. This would result in a sensitivity matrix requiring $\sim 80 \mathrm{~GB}$ in storage, which is impractical to calculate and store, let alone manipulate or decompose.

Each data point, however, is sensitive to a very limited number of elements in the 3D model only. In what follows, we have used the survey parameters from the subsequent Bookpurnong case study as an example of the limited spatial extent of the sensitivity. We have used a flight height of $37 \mathrm{~m}$ and a half-space of $200 \mathrm{mS} / \mathrm{m}$ to calculate the sensitivity. Figure 1 shows the percentage of the total response from within a square footprint of varying sizes. Each individual frequency is shown, along with the integrated sensitivity across all frequencies. As we would expect, the figure shows that the higher frequencies have a smaller footprint with almost $100 \%$ of their response coming from within a $200 \mathrm{~m}$ footprint. This agrees well with Liu and Becker (1990), who suggested that at the inductive limit, the footprint would be $\sim 140 \mathrm{~m}$. The highest frequency is close to the inductive limit, and $\sim 90 \%$ of the response comes from within a $140 \mathrm{~m}$ footprint at this frequency. The integrated sensitivity shows that over $95 \%$ of the total instrument response comes from within a $200 \mathrm{~m}$ footprint shown on Figure 2. This demonstrates also how small the footprint is compared to the entire $3 \mathrm{D}$ inversion domain.
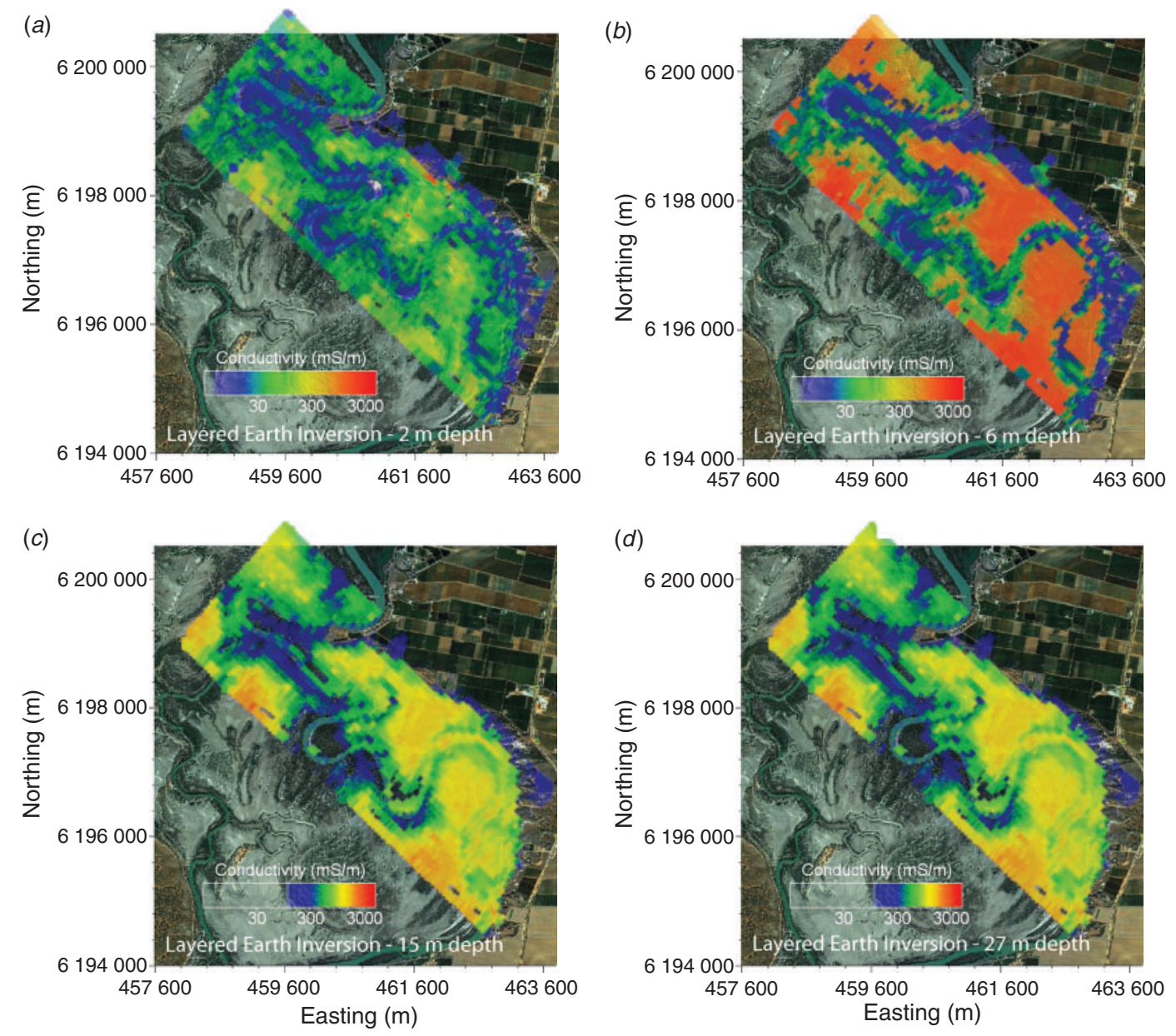

Fig. 3. Horizontal cross-sections of conductivity at different depths obtained from the layered earth inversion result for the Bookpurnong RESOLVE data: (a) $2 \mathrm{~m}$ depth, (b) $6 \mathrm{~m}$ depth, (c) $15 \mathrm{~m}$ depth and (d) $27 \mathrm{~m}$ depth. 
For 3D inversion, the sensitivity matrix can be constructed as a sparse matrix with memory and computational requirements reduced by several orders of magnitude. The number of nonzero elements in each row of the sensitivity matrix is just the number of elements within each footprint (in an order of hundreds or thousands) rather than the total number of elements in the domain (hundreds of thousands). There is no need to calculate the Green's body-to-receiver tensors and background fields for elements which are outside the footprint. Moreover, since the background model is horizontally layered, the body-to-body Green's tensors are horizontally invariant. Hence, the electric Green's tensors are identical for each footprint domain. This means, they are calculated only once for a single footprint and then translated over the entire inversion domain, vastly speeding up the computation and increasing memory efficiency. The transmitter positions and their footprints need not correspond with element positions or centres. In practice, this indexing can be generalised to include multiple channels, variable footprint size, and arbitrary model discretisation.

\section{Case study - Bookpurnong}

\section{Geological background}

The Bookpurnong Irrigation District is located along the Murray River, $\sim 12 \mathrm{~km}$ upstream from the township of Loxton, South Australia. This area has been the focus of various trials to manage a decline in vegetation; largely in response to floodplain salinisation from groundwater discharge in combination with decreased flooding frequency, permanent weir pool levels and recent drought. The result has been the accumulation and concentration of salt within floodplain soils, and an increase in salt loads to the river.

Various ground-based, river-borne and AEM methods have been deployed with the intent of mapping the distribution of salinity in the floodplain soils and groundwater to discover patterns and processes relating to groundwater evapotranspiration and flow across the salinising floodplains. We refer the readers to Munday et al. $(2006,2007)$ for a more detailed description of the geology, hydrology, and various river, borehole, ground and AEM surveys relating to the Bookpurnong area. We will constrain ourselves only to a brief overview of the area, and focus on the 3D inversion of AEM data which has been the subject of previous 1D analyses (e.g. Munday et al., 2006, 2007; Brodie and Sambridge, 2009; Viezzoli et al., 2009; Christensen et al., 2010).

The floodplain sediments consist of the Coonambidgal Clay, with a thickness between $3 \mathrm{~m}$ and $7 \mathrm{~m}$, overlying the Monoman Formation sands, themselves $\sim 7 \mathrm{~m}$ to $10 \mathrm{~m}$ thick. These sediments occupy the Murray Trench which cuts into the Loxton-Parilla Sands, which are up to $35 \mathrm{~m}$ thick in the area. The Loxton-Parilla Sands crop out in the adjacent cliffs, and are covered by a layer of the Woorinen Sands over Blanchetown Clay; each $\sim 2 \mathrm{~m}$ thick. Regional groundwater salinity in the Loxton Sands and Monoman Formation ranges between $30000 \mathrm{mg} / \mathrm{L}$ and $40000 \mathrm{mg} / \mathrm{L}$, with the high salinities commonly found on the floodplain resulting from evaporate concentration. Groundwater
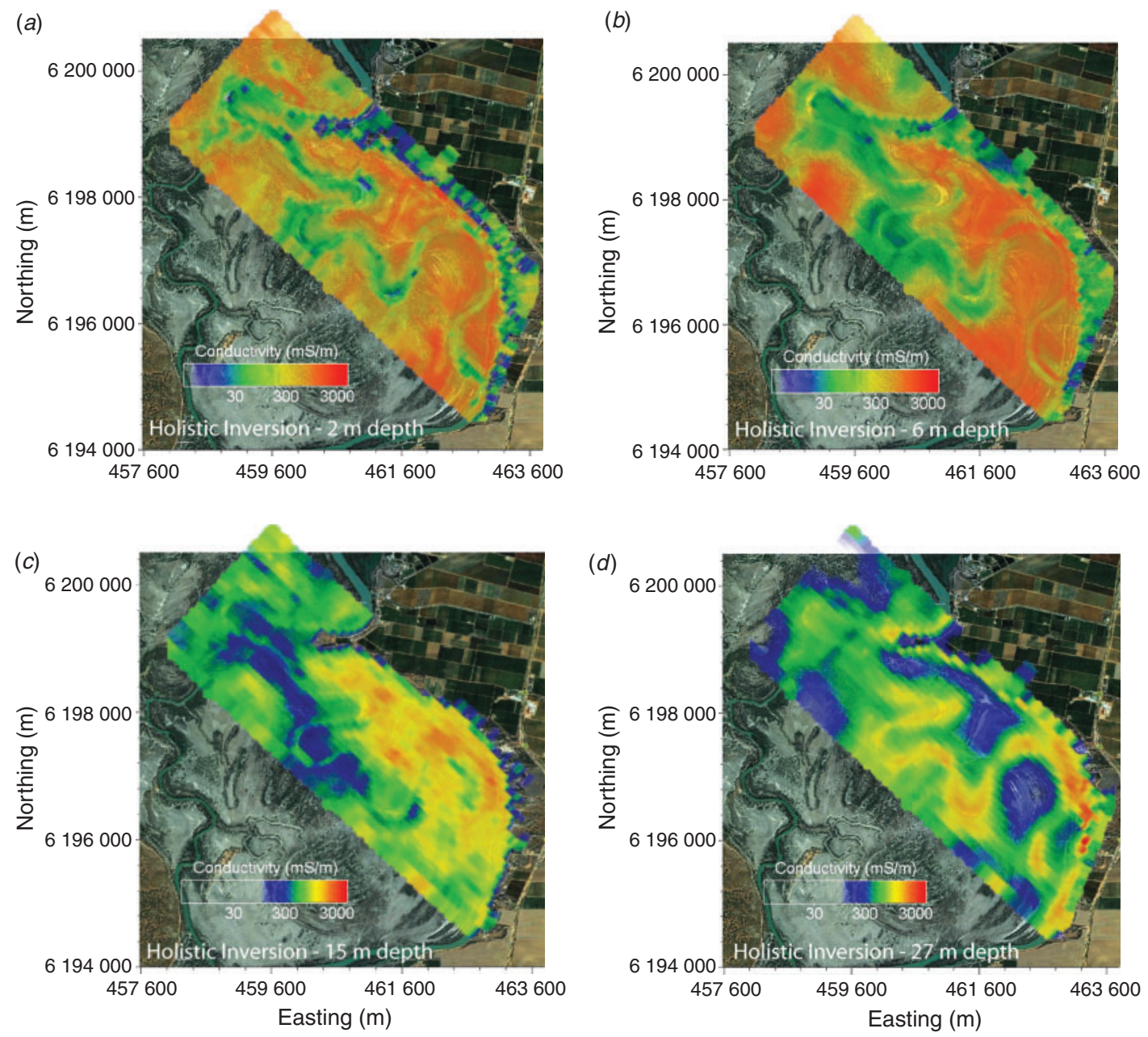

Fig. 4. Horizontal cross-sections of conductivity at different depths obtained from the holistic inversion result for the Bookpurnong RESOLVE data: (a) $2 \mathrm{~m}$ depth, (b) $6 \mathrm{~m}$ depth, (c) $15 \mathrm{~m}$ depth and (d) $27 \mathrm{~m}$ depth. 
levels are $2 \mathrm{~m}$ to $6 \mathrm{~m}$ below the floodplain, and $20 \mathrm{~m}$ to $30 \mathrm{~m}$ below the surface in the adjacent highland areas.

\section{AEM inversion}

In both July 2005 and August 2008, the area was flown with the RESOLVE frequency-domain helicopter system. We analysed the August 2008 data. The RESOLVE system was configured with six operating frequencies: $390,1798,8177,39460$ and $132700 \mathrm{~Hz}$ horizontal coplanar and $3242 \mathrm{~Hz}$ vertical coaxial. The transmitter-receiver separation was $7.91 \mathrm{~m}$ for the five horizontal coplanar coil sets, and $8.99 \mathrm{~m}$ for the single vertical coaxial coil set. This consisted of $\sim 45000$ stations flown as 146 line $\mathrm{km}$ of data. This survey was distributed over 26 lines oriented in a NW-SE direction with $100 \mathrm{~m}$ line spacing, and seven tie lines (see Figure 2). The survey was flown with a nominal bird height of $\sim 45 \mathrm{~m}$ because of the presence of trees along the river bank. Every fifth data point from the survey was used for both layered earth and 3D inversion, resulting in an along line spacing between stations of $\sim 15 \mathrm{~m}$. This was done to minimise data redundancy. In what follows, all misfits are quoted as a point symmetric root mean square error. For layered earth inversion, these are quoted per station, and for $3 \mathrm{D}$ inversion, are quoted for the entire survey.

The layered earth inversion, run with AirBeo (Raiche et al., 2007), was given a starting model of four layers and we inverted for resistivity and thickness of each layer. The upper layer was constrained to a thickness between $2 \mathrm{~m}$ and $10 \mathrm{~m}$, and the other layers were contained to thicknesses between $5 \mathrm{~m}$ and $15 \mathrm{~m}$.
Generally, the layered earth inversion for each station converged to a misfit less than $10 \%$.

For our 3D inversion, we discretised the domain into $25 \mathrm{~m} \times 25 \mathrm{~m}$ horizontal elements with 10 elements in the $z$ direction, ranging in thickness from $2 \mathrm{~m}$ near the surface to $11 \mathrm{~m}$ in the deepest elements. This discretisation created a 309000 element inversion domain superimposed on a $200 \mathrm{mS} / \mathrm{m}$ halfspace background conductivity model. With this discretisation and the $200 \mathrm{~m}$ footprint chosen, each transmitter is only sensitive to 640 model elements, instead of the full 309000 as would be required without the footprint approach. This leads to approximately a 500 times reduction in storage requirements. All coplanar and coaxial channels were jointly inverted for a total of $\sim 35000$ data points. For regularisation, we used a minimum norm stabiliser which minimises departures from the a priori model; in this case, a homogeneous half-space. The inversion converged to a final misfit of $10 \%$ after starting from an initial misfit of $60 \%$. The channels were weighted by the error levels described in Table 1. Our 3D inversion required $45 \mathrm{~min}$ on a 64-bit Linux workstation using five $2.4 \mathrm{GHz}$ processors and 24 GB of RAM. The pre-computation of the Green's tensors and background fields required $25 \mathrm{~min}$, so additional inversion runs required only $20 \mathrm{~min}$.

\section{Interpretation}

For comparison, the same RESOLVE data had been independently inverted using an approximate layered earth inversion (Christensen, 2008; Christensen et al., 2010),
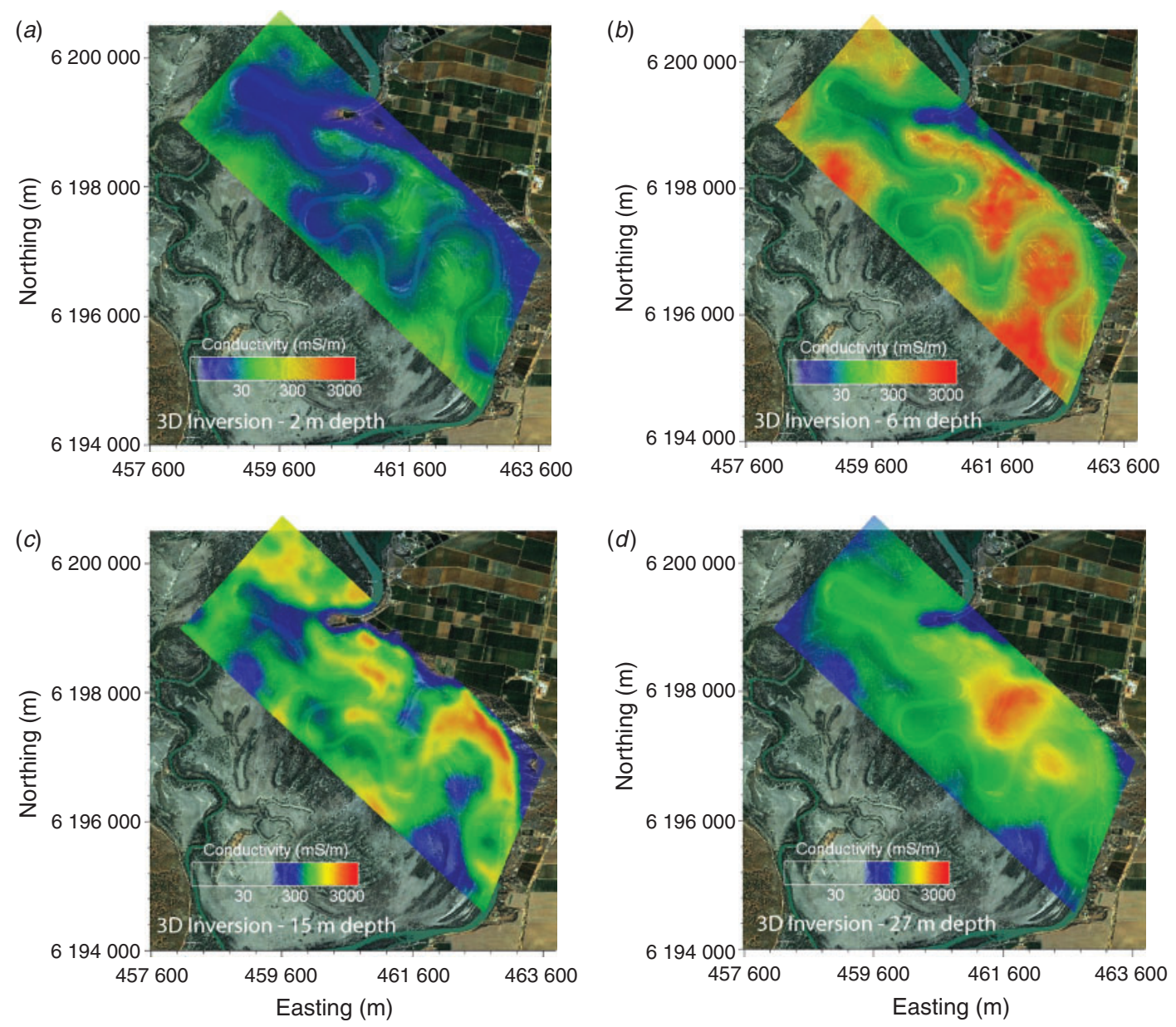

Fig. 5. Horizontal cross-sections of conductivity at different depths obtained from the 3D inversion result for the Bookpurnong RESOLVE data: (a) $2 \mathrm{~m}$ depth, (b) $6 \mathrm{~m}$ depth, (c) $15 \mathrm{~m}$ depth and (d) $27 \mathrm{~m}$ depth. 
laterally constrained inversion (Viezoli et al., 2009) and holistic inversion (Brodie and Sambridge, 2006, 2009). Only the holistic inversion results were made available to us (T. Munday, pers. comm.). As was discussed previously, we also inverted the data using a layered earth inversion (AirBeo; Raiche et al., 2007). The results obtained from all the 1D methods are generally consistent, whereby broad variations in conductivity attributed to salinity across the floodplains and in the river sediments are observed. The $1 \mathrm{D}$ inversion results were re-gridded to a $25 \mathrm{~m}$ horizontal element size, the same as the 3D inversion. For both the layered earth and 3D inversions, the data delivered by Fugro Airborne Surveys were not scaled. The holistic inversion, by definition, scales the data by calibration factors, effectively meaning that it modifies both the data and the model during inversion. We argue that such calibration factors are unnecessary for our 3D inversion given they effectively modify the data such that it can be satisfied by $1 \mathrm{D}$ modelling. In what follows, this data calibration could potentially explain the difference in near-surface conductivities between the layered earth and 3D inversion results, compared to the holistic inversion results.

Figures 3-5 show the conductivity cross-sections for depths of $3 \mathrm{~m}, 6 \mathrm{~m}, 15 \mathrm{~m}$ and $30 \mathrm{~m}$ for layered earth inversion, holistic inversion and 3D inversion, respectively. In all three figures, the general location of the Murray River, the more conducting sediments surrounding the Murray River, and the transition to the highlands can all be seen. The layered earth and 3D inversion results agree very well about the conductivity of the upper layer, while the holistic inversion shows conductivities that are approximately an order of magnitude higher. We suggest this is due to the calibration factors introduced to modify both the data and the model during holistic inversion. At $6 \mathrm{~m}$ depth, the layered earth, holistic and 3D inversions all agree quite well. At $15 \mathrm{~m}$, the holistic and 3D inversions agree well, while the layered earth inversion retains many of the conductivity features from the layers above. The gaining sections of the Murray River where the saline water is being pushed up into the bottom of the river channel and the flushed section are visible in both the holistic and 3D inversion. However, the $3 \mathrm{D}$ inversion is spatially more coherent and shows sharper conductivity bounds than the holistic inversion. At $30 \mathrm{~m}$, the holistic inversion has ghostly artefacts of the Murray River (possibly due to overregularisation), whereas the $3 \mathrm{D}$ inversion has a coherent conductive feature that correlates with groundwater influx. In all cross-sections, the 3D inversion shows sharper lateral

A

(a) Layered earth inversion

$A^{\prime}$

Conductivity $(\mathrm{mS} / \mathrm{m})$

\section{(a) Layered earth inversion}

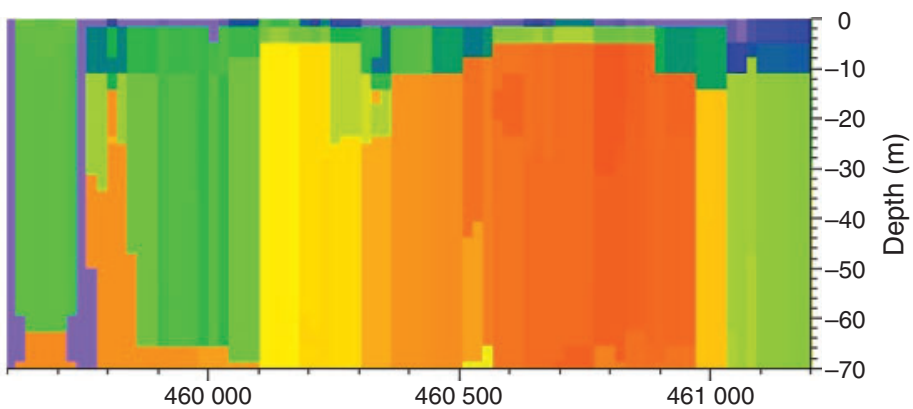

(b) Holistic inversion
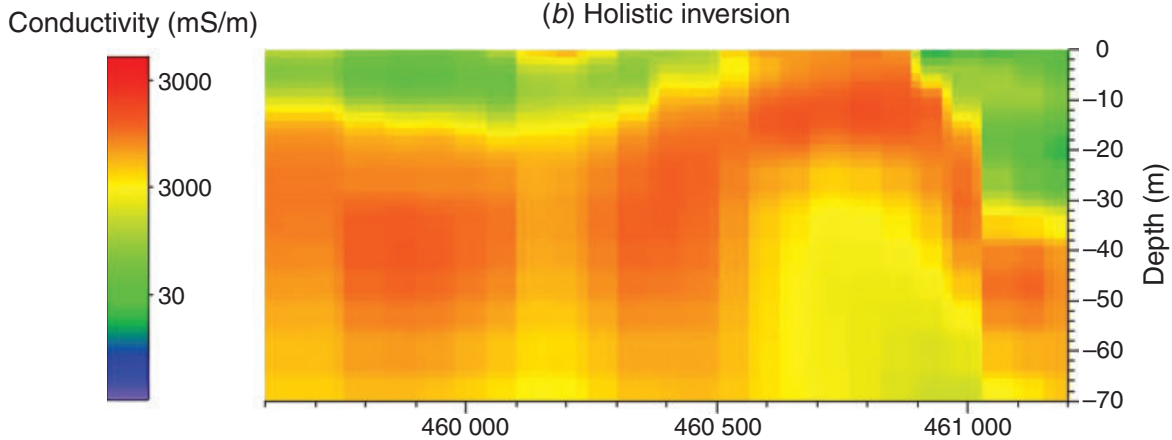

Conductivity $(\mathrm{mS} / \mathrm{m})$

(c) 3D inversion
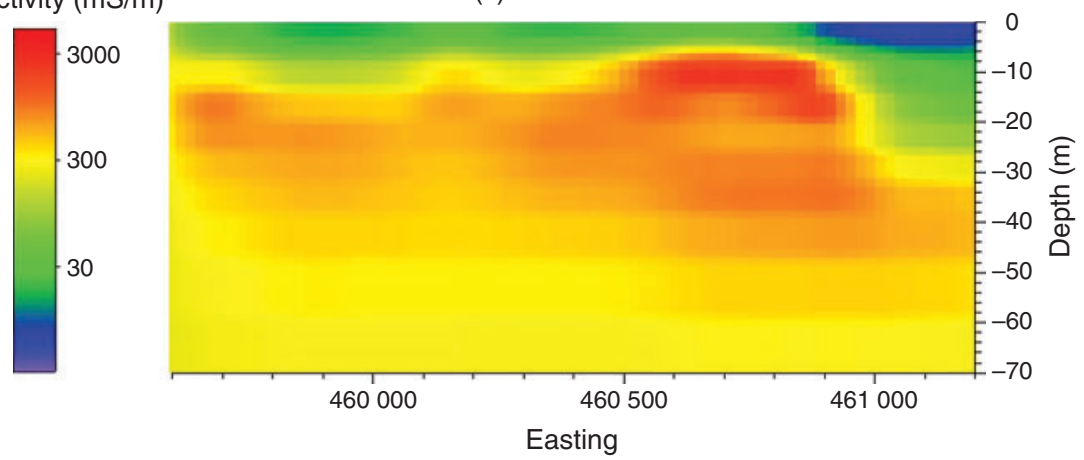

Fig. 6. Vertical cross-sections of conductivity along profile A-A', as shown in Fig. 2, for $(a)$ layered earth inversion, (b) holistic inversion, and (c) 3D inversion. 
B

Conductivity $(\mathrm{mS} / \mathrm{m})$
$\mathrm{B}^{\prime}$

(a) Layered earth inversion

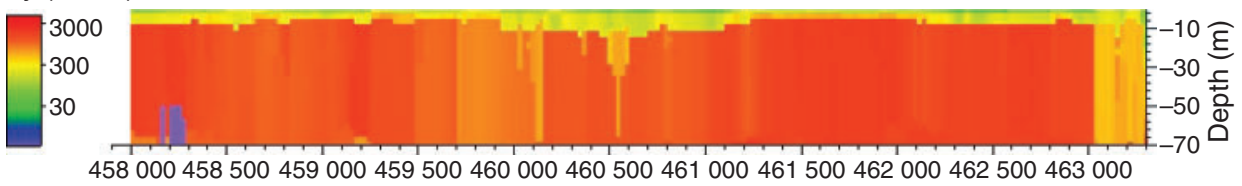

Conductivity $(\mathrm{mS} / \mathrm{m})$

(b) Holistic inversion

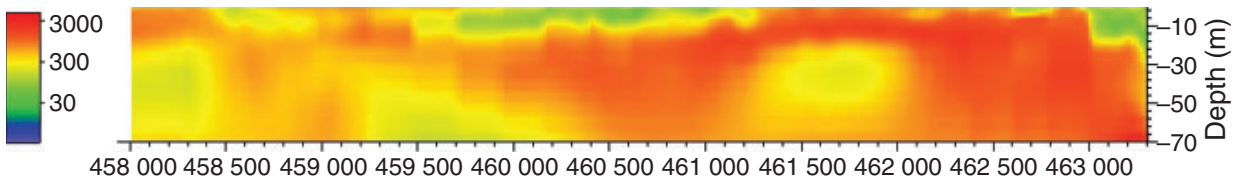

458000458500459000459500460000460500461000461500462000462500463000

Conductivity $(\mathrm{mS} / \mathrm{m})$

(c) 3D inversion

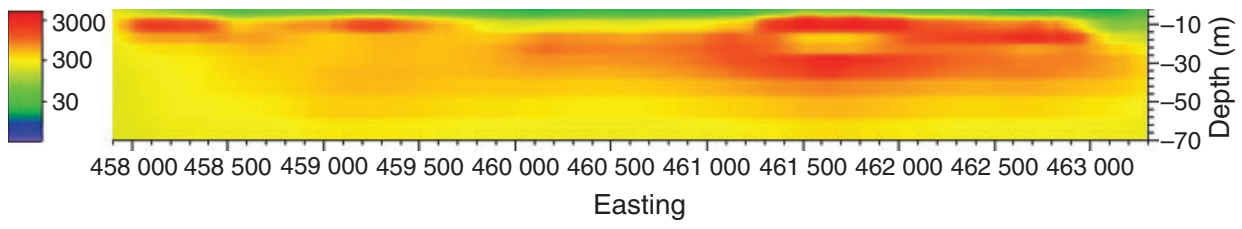

Fig. 7. Vertical cross-sections of conductivity along profile B-B', as shown in Fig. 2, for $(a)$ layered earth inversion, (b) holistic inversion, and (c) 3D inversion.

boundaries and produces a more coherent image of the 3D conductivity distribution than either of the $1 \mathrm{D}$ methods.

Figure 6 shows a vertical cross-section of the conductivity along the line $\mathrm{A}-\mathrm{A}^{\prime}$, the location of which is given in Figure 2. This line corresponds to geological sections presented in Munday et al. (2006). In Figure 6, all sections show a conductivity anomaly $c$. $460750 \mathrm{E}$ which corresponds to the build up of a saline groundwater mound. Other than to produce a conductivity anomaly, the layered earth inversion fails to yield any structural information on the conductivity distribution. The holistic inversion produces a single conductive layer, whereas our 3D inversion recovers layers that may correlate to different sand formations. Compared to the geological sections of Munday et al. (2006), the holistic inversion fails to recover the relatively high resistivity fresh irrigation water influx and the highlands to the right of profile $\mathrm{A}-\mathrm{A}^{\prime}$, whereas $3 \mathrm{D}$ inversion recovers this feature. Both layered earth and holistic inversions show a conductivity discontinuity typical of $1 \mathrm{D}$ inversion at $461000 \mathrm{E}$ that is not apparent in the $3 \mathrm{D}$ inversion. When fitted to topography, this particular discontinuity coincides with the floodplain-highland boundary. This suggests that the 3D conductivity structure is better modelled by $3 \mathrm{D}$ methods when there are changes in topography.

Figure 7 shows a vertical cross-section of the conductivity along the line B-B' as shown in Figure 2. Again, this line corresponds to geological sections presented in Munday et al. (2006). In Figure 7, the 3D inversion discriminates layering along profile B-B' not apparent in either the layered earth or holistic inversions. The main difference between the layered earth, holistic and 3D inversion results is the deeper conductivity distribution. From sensitivity analysis, it is known that over $95 \%$ of the total RESOLVE response is due to the top $50 \mathrm{~m}$ of the Earth model, so the data have virtually no sensitivity to these deeper sections of the models. Hence, confidence in the models below $50 \mathrm{~m}$ must be questioned. The $3 \mathrm{D}$ inversion has minimum deviation from the initial model of $500 \mathrm{mS} / \mathrm{m}$ at depths greater than $50 \mathrm{~m}$, whereas the layered earth and holistic inversions propagate the last resolved conductivity to depths greater than $50 \mathrm{~m}$.
Table 2. Run times per station (normalised by number of processors) for selected airborne electromagnetic inversion algorithms.

\begin{tabular}{|c|c|}
\hline Inversion method & Time per station (s) \\
\hline $\begin{array}{l}\text { 1D non-linear inversion } \\
\text { (Christensen, 2008) }\end{array}$ & 3.38 \\
\hline 3D inversion & 1.75 \\
\hline $\begin{array}{l}\text { 1D approximate inversion } \\
\text { (Christensen, 2008) }\end{array}$ & 0.22 \\
\hline $\begin{array}{l}\text { 1D non-linear inversion } \\
\text { (AirBeo; Raiche et al., 2007) }\end{array}$ & 0.08 \\
\hline
\end{tabular}

The main argument for various 1D interpretations for AEM data is that $3 \mathrm{D}$ inversion is not practical. Table 2 shows the $3 \mathrm{D}$ inversion run time per station relative to different $1 \mathrm{D}$ algorithms for which runtimes on the Boopurnong RESOLVE data have been published or are available. We conclude that $3 \mathrm{D}$ inversion has runtimes comparable to those of $1 \mathrm{D}$ inversion methods.

\section{Conclusions}

In the past, 3D inversion of AEM data was believed to be impractical. As a result, emphasis was placed on an improvement of the available 1D methods rather than on the pursuit of 3D inversion. A variety of competing $1 \mathrm{D}$ methods were developed and have been used to interpret AEM data. However, 1D methods have difficulties when applied to 3D geology. In this paper, we have shown that our developed 3D inversion code has comparable runtimes to $1 \mathrm{D}$ methods using similar computational resources. Therefore, 3D AEM inversion can be made practical. We have developed a robust 3D inversion strategy for entire AEM surveys based on a moving footprint. Our implementation is based on the 3D integral equation method for computing data and sensitivities, as well as the re-weighted regularised conjugate gradient method for minimising the parametric functional. We thus avoid the need for approximations in our modelling and inversion algorithms. Our 3D inversion strategy vastly speeds up the inversion process compared to traditional $3 \mathrm{D}$ inversion. The software we have developed can be used to invert entire surveys of 
frequency-domain AEM data within hours on a multi-processor workstation. We are now distributing our 3D AEM inversion software on massively parallel architectures in order to further reduce the runtimes. Our approach can be naturally extended to time-domain AEM inversion. We will discuss this further in a subsequent paper.

\section{Acknowledgements}

The authors acknowledge TechnoImaging for supporting this research and giving permission to publish. The RESOLVE data were financed in part by the South Australian CNMR Board Project 054127: The application of airborne geophysics to the prediction of groundwater recharge and floodplain salinity management, and the CSIRO National Flagship program Water for a Healthy Country through the Regional Water theme. We thank Dr Andrew Fitzpatrick for providing the RESOLVE data, and Dr Tim Munday for providing the holistic inversion results that were used to compare our 3D model. We thank Elena Zhdanov, Editor-in-Chief Dr Mark Lackie, Associate Editor Dr Julian Vrbancich, and reviewers Drs Tim Munday and Peter Wolfgram, for their helpful suggestions which improved the manuscript.

\section{References}

Auken, E., Chistiansen, A. V., Jacobsen, B. H., Foged, N., and Sorensen, K. L., 2005, Piece-wise 1D laterally constrained inversion of resistivity data: Geophysical Prospecting, 53, 497-506. doi:10.1111/j.13652478.2005.00486.x

Beamish, D., 2003, Airborne EM footprints: Geophysical Prospecting, 51, 49-60. doi:10.1046/j.1365-2478.2003.00353.x

Brodie, R., and Sambridge, M., 2006, A holistic approach to inversion of frequency-domain airborne EM data: Geophysics, 71, G301-G312. doi:10.1190/1.2356112

Brodie, R., and Sambridge, M., 2009, Holistic inversion of frequency-domain airborne electromagnetic data with minimal prior information: Exploration Geophysics, 40, 8-16. doi:10.1071/EG08110

Chen, J., and Raiche, A., 1998, Inverting AEM data using a damped eigenparameter method: Exploration Geophysics, 29, 128-132. doi:10.1071/EG998128

Christensen, N. B., 2008, Fast approximate 1D inversion of FDHEM data Bookpurnong, South Australia: CSIRO Exploration and Mining Report Number P2008/1251.

Christensen, N. B., Fitzpatrick, A., and Munday, T., 2010, Fast approximate inversion of frequency-domain electromagnetic data: Near Surface Geophysics, 8, 1-15.

Cox, L. H., and Zhdanov, M. S., 2006, Rapid and rigorous 3D inversion of airborne electromagnetic data: presented at SEG International Exposition and 76th Annual Meeting, New Orleans.

Cox, L. H., and Zhdanov, M. S., 2007, Large scale 3D inversion of HEM data using a moving footprint: presented at SEG International Exposition and 77th Annual Meeting, San Antonio.

Cox, L. H., and Zhdanov, M. S., 2008, Advanced computational methods for rapid and rigorous $3 \mathrm{D}$ inversion of airborne electromagnetic data: Communications in Computational Physics, 3, 160-179.

Combrinck, M., 2008, Calculation of conductivity and depth correction factors for the S-layer differential transform: Exploration Geophysics, 39, 133-138. doi:10.1071/EG08014

Ellis, R. G., 1995, Joint 3D EM inversion: International Symposium on ThreeDimensional Electromagnetics, Expanded Abstracts, 307-323.

Ellis, R. G., 1998, Inversion of airborne electromagnetic data: Exploration Geophysics, 29, 121-127. doi:10.1071/EG998121

Ellis, R. G., 2002, Electromagnetic inversion using the QMR-FFT fast integral equation method: presented at SEG International Exposition and 72nd Annual Meeting, Salt Lake City.

Farquharson, C. G., Oldenburg, D. W., and Routh, P. S., 2003, Simultaneous $1-D$ inversion of loop-loop electromagnetic data for magnetic susceptibility and electrical conductivity: Geophysics, 68, 1857-1869. doi: $10.1190 / 1.1635038$

Fullagar, P. K., and Reid, J. E., 2001, Emax conductivity-depth transformation of airborne TEM data: presented at ASEG 15th Geophysical Conference and Exhibition, Brisbane.
Hohmann, G. W., 1975, Three-dimensional induced polarization and electromagnetic modelling: Geophysics, 40, 309-324. doi:10.1190/ 1.1440527

Huang, H., and Fraser, D. C., 2002, Dielectric permittivity and resistivity mapping using high frequency, helicopter-borne EM data: Geophysics, 67, 727-738. doi:10.1190/1.1484515

Hursán, G., and Zhdanov, M. S., 2002, Contraction integral equation method in three-dimensional electromagnetic modelling: Radio Science, 37. doi:10.1029/2001RS002513

Liu, G., and Becker, A., 1990, Two-dimensional mapping of sea-ice keels with airborne electromagnetic: Geophysics, 55, 239-248. doi:10.1190/ 1.1442832

Macnae, J., King, A., Stolz, N., Osmakoff, A., and Blaha, A., 1998, Fast AEM data processing and inversion: Exploration Geophysics, 29, 163-169. doi:10.1071/EG998163

Munday, T., Fitzpatrick, A., Doble, R., Berens, V., Hatch, M., and Cahill, K., 2006, The combined use of air, ground and 'in river' electromagnetic in defining spatial processes of salinisation across ecologically important floodplain areas - Lower River Murray, SA. In Fitzpatrick, R. W. and Shand, P. (Eds), Proceedings of the CRC LEME Regolith Symposium, 249-255.

Munday, T., Fitzpatrick, A., Reid, J., Berens, V., and Sattel, D., 2007, Frequency and/or time-domain HEM systems for defining floodplain processes linked to the salinisation along the Murray River: presented at ASEG 19th Geophysical Conference and Exhibition, Perth.

Nabighian, M. N., 1979, Quasi-static transient response of a conducting halfspace - An approximate representation: Geophysics, 44, 1700-1705. doi:10.1190/1.1440931

Raiche, A. P., 1974, An integral equation approach to three-dimensional modelling: Geophysical Journal of the Royal Astronomical Society, 36, 363-376.

Raiche, A., Annetts, D., and Sugeng, F., 2001, EM target response in complex hosts: presented at ASEG 15th Geophysical Conference and Exhibition, Brisbane.

Raiche, A., Wilson, G., and Sugeng, F., 2006, Practical 3D EM inversion for thin sheet structures: presented at Australian Earth Science Convention, Melbourne.

Raiche, A., Sugeng, F., and Wilson, G., 2007, Practical 3D EM inversion P223F software suite: presented at ASEG 19th Geophysical Conference and Exhibition, Perth.

Reid, J. E., and Macnae, J. C., 1998, Comments on the electromagnetic "smoke ring" concept: Geophysics, 63, 1908-1913. doi:10.1190/ 1.1444483

Reid, J. E., Pfaffling, A., and Vrbancich, J., 2006, Airborne electromagnetic footprints in 1D earths: Geophysics, 71, G63-G72. doi:10.1190/ 1.2187756

Sattel, D., 2005, Inverting airborne electromagnetic (AEM) data using Zohdy's method: Geophysics, 70, G77-G85. doi:10.1190/ 1.1990217

Tartaras, E., and Beamish, D., 2005, Laterally constrained inversion of fixed-wing frequency-domain AEM data: presented at 12th European Meeting of Environmental and Near Surface Geophysics, Helsinki.

Tartaras, E., Zhdanov, M. S., Wada, K., Saito, A., and Hara, T., 2000, Fast imaging of TDEM data based on S-inversion: Journal of Applied Geophysics, 43, 15-32. doi:10.1016/S0926-9851(99)00030-0

Vallée, M. A., and Smith, R. S., 2009, Inversion of airborne time-domain electromagnetic data to a 1D structure using lateral constraints: Near Surface Geophysics, 7, 63-71.

Viezzoli, A., Auken, E., and Munday, T., 2009, Spatially constrained inversion for quasi 3D modelling of airborne electromagnetic data - an application for environmental assessment in the Lower Murray Region of South Australia: Exploration Geophysics, 40, 173-183. doi:10.1071/ EG08027

Weidelt, P., 1975, EM induction in three-dimensional structures: Journal of Geophysics, 49, 60-74.

Wilson, G. A., Raiche, A. P., and Sugeng, F., 2006, 2.5D inversion of airborne electromagnetic data: Exploration Geophysics, 37, 363-371. doi:10.1071/EG06363

Wilson, G. A., Cox, L. H., and Zhdanov, M. S., 2010, Practical 3D inversion of entire airborne electromagnetic surveys: Preview, 146, 29-33. 
Wolfgram, P., and Karlik, G., 1995, Conductivity-depth transform of GEOTEM data: Exploration Geophysics, 26, 179-185. doi:10.1071/ EG995179

Wolfgram, P., and Golden, H., 2001, Airborne EM applied to sulphide nickelexamples and analysis: Exploration Geophysics, 32, 136-140. doi:10.1071/EG01136

Xiong, Z., 1992, Electromagnetic modeling of 3D structures by the method of system iteration using integral equations: Geophysics, 57, 1556-1561. doi:10.1190/1.1443223

Zhang, Z., 2003, 3D resistivity mapping of airborne EM data: Geophysics, 68 , 1896-1905. doi:10.1190/1.1635042

Zhdanov, M. S., 2002, Geophysical Inverse Theory and Regularization Problems: Elsevier.

Zhdanov, M. S., 2009, Geophysical Electromagnetic Theory and Methods: Elsevier.
Zhdanov, M. S., and Chernyavskiy, A., 2004, Rapid three-dimensional inversion of multi-transmitter electromagnetic data using the spectral Lanczos decomposition method: Inverse Problems, 20, S233-S256. doi:10.1088/0266-5611/20/6/S14

Zhdanov, M. S., and Tartaras, E., 2002, Three-dimensional inversion of multitransmitter electromagnetic data based on the localized quasi-linear approximation: Geophysical Journal International, 148, 506-519. doi:10.1046/j.1365-246x.2002.01591.x

Zhdanov, M. S., Pavlov, D., and Ellis, R. G., 2002, Localized S-inversion for time-domain electromagnetic data: Geophysics, 67, 1115-1125. doi:10.1190/1.1500372

Manuscript received on 18 January 2010; accepted 19 November 2010. 